\title{
Reverse shape selectivity in the adsorption of hexane and xylene isomers in MOF UiO-66
}

\author{
Patrick S. Bárcia ${ }^{\text {a,b,* }}$, Daniela Guimarães ${ }^{\text {b }}$, Patrícia A.P. Mendes ${ }^{\text {b }}$, José A.C. Silva ${ }^{\text {, }}$, Vincent Guillerm ${ }^{\text {, }}$, \\ Hubert Chevreau $^{c}$, Christian Serre ${ }^{c}$, Alírio E. Rodrigues ${ }^{a}$

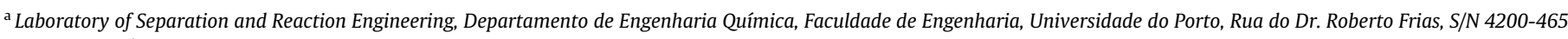 \\ Porto, Portugal \\ ${ }^{\mathrm{b}}$ Escola Superior de Tecnologia e Gestão, Instituto Politécnico de Bragança, Apartado 1134, 5301-857 Bragança, Portugal \\ ${ }^{\mathrm{c}}$ Institut Lavoisier (UMR CNRS 8180), Université de Versailles Saint-Quentin-en-Yvelines, 45 avenue des Etats-Unis, 78035 Versailles cedex, France
}

\section{A R T I C L E I N F O}

\section{Article history:}

Received 23 June 2010

Received in revised form 12 October 2010

Accepted 13 October 2010

Available online 20 October 2010

\section{Keywords:}

Adsorption

Fixed-bed

Vapor mixture

Reverse shape selectivity

Metal-organic framework

\begin{abstract}
A B S T R A C T
An adsorption study of hexane and xylene isomers mixtures was addressed in a rigid zirconium terephthalate UiO-66 (UiO for University of Oslo) with octahedral and tetrahedral cavities of free diameter close to $1.1 \mathrm{~nm}$ and $0.8 \mathrm{~nm}$, respectively. Multicomponent equimolar breakthrough experiments show that the adsorption hierarchy of structural isomers in UiO-66 is opposite to the one observed in conventional adsorbents. For hexane isomers, it was found that the amount adsorbed increases with the degree of branching, being 2,2-dimethylbutane (22DMB) and 2,3-dimethylbutane (23DMB) the more retained molecules. Regarding the xylene isomers, the results show that the adsorption of the bulkier ortho-xylene (oX) is favoured compared to its homologues. The structural similarity between MOF UiO-66 and zeolite MCM-22 suggests that the reverse shape selectivity observed in the adsorption of hexane and xylene isomers might be attributed to the rotational freedom of the molecules inside the small cavities.
\end{abstract}

(C) 2010 Elsevier Inc. All rights reserved.

\section{Introduction}

The metal-organic framework (MOF) UiO-66 is a zirconiumbased metal-organic framework (MOF) built up from hexamers of eight coordinated $\mathrm{ZrO}_{6}(\mathrm{OH})_{2}$ polyhedra and 1,4-benzene-dicarboxylate (BDC) linkers. Its cubic rigid 3D pores structure consists of an array of octahedral cavities of diameter $1.1 \mathrm{~nm}$, and tetrahedral cavities of diameter $0.8 \mathrm{~nm}$, as shown in Fig. 1. Accessibility to the cavities is ensured through microporous triangular windows of free diameter within the $0.5-0.7 \mathrm{~nm}$ range. UiO-66 possesses also a good chemical resistance toward solvents like water, acetone, benzene and dimethylformamide (DMF), and a suitable mechanical ( $>10$ tons $/ \mathrm{cm}^{2}$ ) and thermal stability (up to $773 \mathrm{~K}$ ) for industrial applications [1].

MOFs offer the possibility to be tuned varying their pore size and geometry for specific applications [2-4]. This feature has the potential for making a significant impact in the development of adsorption-based separation processes for structural isomers molecules, which generally relies on the subtle matching of the size and shape of the adsorbates and the adsorbent micropores. Such

\footnotetext{
* Corresponding author at: Laboratory of Separation and Reaction Engineering, Departamento de Engenharia Química, Faculdade de Engenharia, Universidade do Porto, Rua do Dr. Roberto Frias, S/N 4200-465 Porto, Portugal. Tel.: +351 27330 3191; fax: +351273 313051 .

E-mail address: patrick.barcia@ipb.pt (P.S. Bárcia).
}

mechanism, known in the literature as shape-selective effect, commonly favours the adsorption of molecules with smaller kinetic diameter, excluding the bulkier ones from accessing the micropores [5]. Good examples of its practical application are the advanced recycle schemes for light naphtha isomerisation, commercialized by UOP (Total Isomerization Process), and Axens (IPSORB process). These processes are used to boost the research octane number (RON) of the gasoline by separating the low RON linear alkanes in the $C_{5} / C_{6}$ range from their bulkier isomers using zeolite 5A molecular sieves [6,7].

In the present study, we explore the particular arrangement of the cavities in the UiO-66 structure, and its unusual adsorption behaviour of hexane and xylene isomers. The retention of alkane and aromatic molecules in MOF UiO-66 is apparently dictated by their ability to rotate freely in the smaller cavity. As a result, bulkier and more compact molecules are preferably adsorbed over their isomers. Such effect was previously reported by Denayer et al. [8,9] for the adsorption of linear and monobranched alkanes in zeolite MCM-22. Unusual separation of $n$-butane from normal hydrocarbons above $\mathrm{C}_{4}$ in a fluorinated MOF with 1D channels, alternating large chambers and narrow windows, have been described by Pan et al. [10] as a case of molecular length selectivity.

The present work represents, as far as our knowledge goes, the first example with MOF material in which the adsorption of isomeric hydrocarbons might be governed by the rotational freedom. 


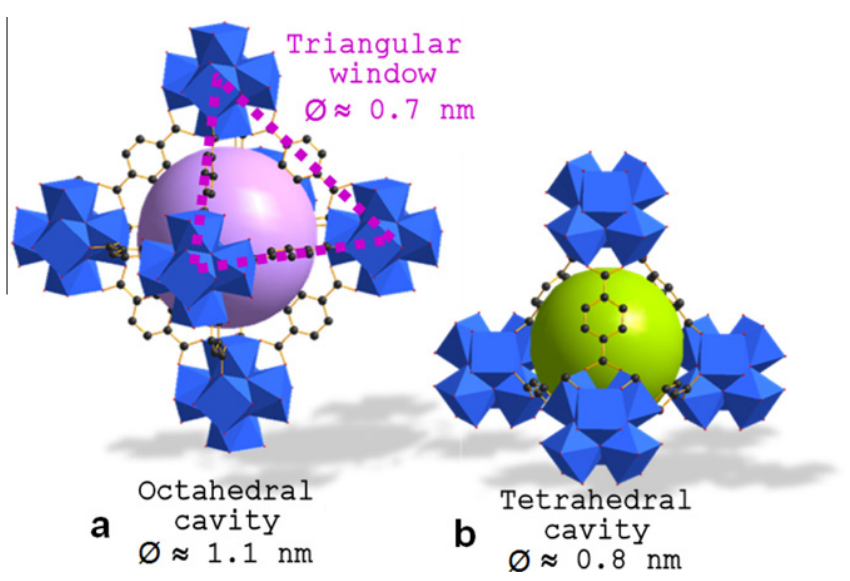

Fig. 1. View of the octahedral (a) and tetrahedral (b) cavities in the highly porous UiO-66. Zirconium polyhedral and carbon atoms are in blue and black, respectively. The free diameters of the octahedral and tetrahedral cavities are represented through purple and green spheres, respectively.

\section{Experimental procedure}

UiO-66 was synthesized at Institut Lavoisier de Versailles (CNRS/Université de Versailles, France), and was later tested at LSRE-IPB (Instituto Politécnico de Bragança, Portugal) for the separation of hexane and xylene isomers vapor mixtures.

The sample was prepared from a mixture of zirconium tetrachloride $\mathrm{ZrCl}_{4}$, terephthalic acid, hydrochloric acid and dimethylformamide in the $25 \mathrm{mmol}$ : $50 \mathrm{mmol}: 50 \mathrm{mmol}: 150 \mathrm{~mL}$ ratio. The slurry was then introduced in a $450 \mathrm{~mL}$ Teflon liner and further introduced in a metallic PARR bomb. The system was heated overnight ( $16 \mathrm{~h}$ ) at $493 \mathrm{~K}$. The resulting white product was filtered off, washed with DMF to remove the excess of unreacted terephthalic acid, then washed again with acetone and dried at room temperature. The sample was finally calcined at $523 \mathrm{~K}$ under vacuum ( 5 mbar) to remove the DMF from the framework. X-ray powder diffractogram (Brüker D5000, $\lambda_{\mathrm{Cu}} \approx 1.5406 \AA$ ) of the sample is in a very good agreement with the one calculated from the published structure. Nitrogen sorption measurements (BelSorp Mini apparatus) gave a BET surface area of $1050(3) \mathrm{m}^{2} / \mathrm{g}$. Both the X-ray pattern and the Nitrogen sorption data are shown in Fig. 2.

Prior to adsorption experiments, UiO-66 in its powdered form was activated to evacuate the excess solvent in the micropores.
The activation was carried out under vacuum (100 mbar) at $473 \mathrm{~K}$, during $12 \mathrm{~h}$. Afterwards, the powder was compacted into disks in an infrared (IR) press; the pressure applied was $650 \mathrm{~kg} /$ $\mathrm{cm}^{2}$ and $2000 \mathrm{~kg} / \mathrm{cm}^{2}$ for the samples used for hexane and xylene isomers, respectively. The disks were then broken into fragments, and sieved to produce a known particle size distribution of agglomerates with size ranging from 0.5 to $1.5 \mathrm{~mm}$. The BET surface area of the agglomerates pressed at $650 \mathrm{~kg} / \mathrm{cm}^{2}$ is $5 \%$ lower than the initial powder surface area $\left(1002(2) \mathrm{m}^{2} \mathrm{~g}^{-1}\right)$. The agglomerates were packed in a stainless steel column with $4.6 \mathrm{~mm}$ internal diameter (the column length was $100 \mathrm{~mm}$ and $80 \mathrm{~mm}$ for the experiments with hexane and xylene isomers, respectively), and connected to a multicomponent breakthrough curve apparatus described elsewhere in the literature [11]. Several adsorption runs were performed with pure $n$ HEX to check up on the permanent microporosity of the adsorbent. For the first runs, a successive increase in the breakthrough time was observed; the reproducibility of the breakthrough curves being achieved from the fourth experiment. This observation suggests that the relatively mild conditions of the activation procedure were not completely efficient regarding the evacuation of the solvent from the microporous structure. Such effect has been interestingly presented in the literature [14].

Multicomponent breakthrough curves with equimolar vapor mixtures of hexane and xylene isomers were measured following the operating procedure described in detail by Bárcia et al. [11,12].

\section{Results}

\subsection{Adsorption of quaternary mixtures of hexane isomers}

To study the potential application of UiO-66 for the separation of $\mathrm{C}_{6}$ isomers, a screening study covering four temperatures in the range $343-473 \mathrm{~K}$, at low pressure $(0.6 \mathrm{kPa})$ and moderate pressure (6.3 kPa) was performed in a fixed-bed. The experimental conditions, and amount adsorbed are listed in Table 1.

As it can be seen from Table 1, the total sorption capacity of UiO-66 at $423 \mathrm{~K}$ is comparable to the ones found for the adsorption of hexanes in the medium pore size zeolite $5 \mathrm{~A}$ ( $10.0 \mathrm{wt} . \%)$ [13], and large pore size zeolite beta ( 8.6 wt.\%) [12].

Surprisingly, branched $\mathrm{C}_{6}$ are more adsorbed in UiO-66 compared to their linear isomers. Additionally, this unusual behaviour is observed in all the range of pressure and temperature herein addressed. This reversed preferential adsorption is obvious from Fig. 3, where the multicomponent breakthrough curves for

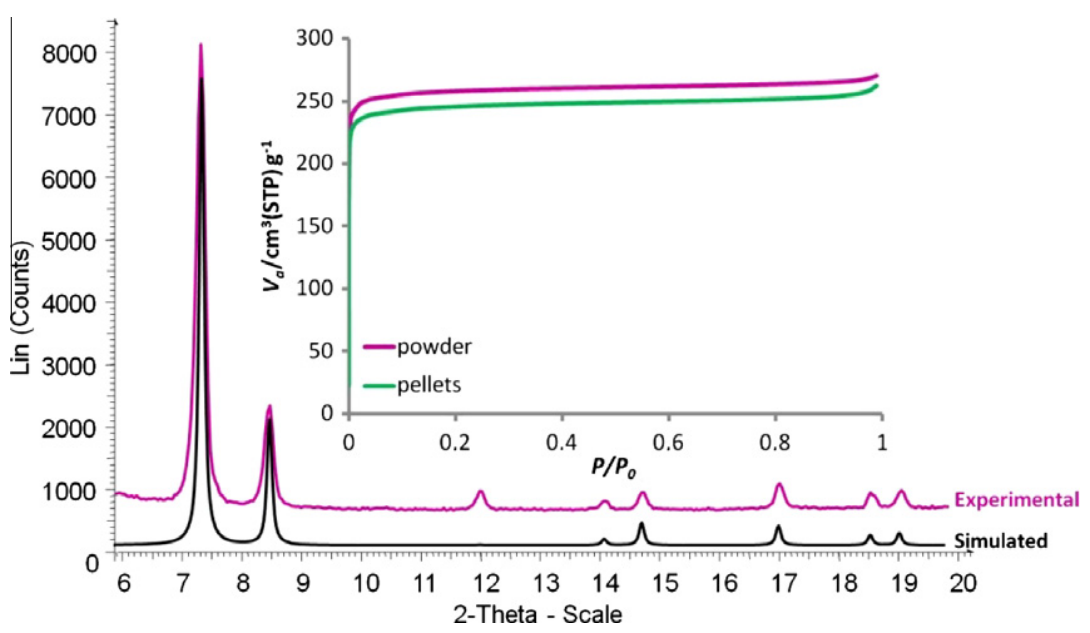

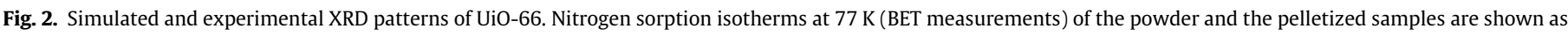
an insert. 
Table 1

Experimental conditions for the equimolar quaternary breakthrough curves of hexane isomers in UiO-66.

\begin{tabular}{|c|c|c|c|c|c|c|c|c|}
\hline \multirow[t]{2}{*}{ Run } & \multirow[t]{2}{*}{$T(\mathrm{~K})$} & \multirow[t]{2}{*}{ Helium flowrate (ml/min) } & \multirow[t]{2}{*}{ Hydrocarbon pressure ( $\mathrm{kPa})$} & \multicolumn{4}{|c|}{ Partial loading (wt.\%) } & \multirow[t]{2}{*}{ Total loading (wt.\%) } \\
\hline & & & & 22DMB & 23DMB & $3 \mathrm{MP}$ & $n$ HEX & \\
\hline UiO66_1a & 343 & 50.0 & 0.6 & 2.81 & 3.04 & 2.64 & 0.87 & 9.35 \\
\hline UiO66_2a & 373 & & & 2.63 & 2.76 & 2.01 & 0.53 & 7.92 \\
\hline UiO66_3a & 423 & & & 1.95 & 1.92 & 1.02 & 0.29 & 5.18 \\
\hline UiO66_4a & 473 & & & 0.61 & 0.57 & 0.40 & 0.20 & 1.78 \\
\hline UiO66_1b & 343 & 15.0 & 6.3 & 2.84 & 3.35 & 2.95 & 1.75 & 10.89 \\
\hline UiO66_2b & 373 & & & 2.97 & 3.26 & 2.70 & 1.46 & 10.39 \\
\hline UiO66_3b & 423 & & & 2.97 & 3.06 & 2.19 & 1.12 & 9.34 \\
\hline UiO66_4b & 473 & & & 2.41 & 2.34 & 1.63 & 0.80 & 7.19 \\
\hline
\end{tabular}

equimolar quaternary mixtures of $\mathrm{C}_{6}$ isomers in UiO-66 are displayed (the experimental conditions are listed in Table 1). It is clear that $n \mathrm{HEX}$ is the less retained molecule in the range of temperature and pressure studied. The large overshoot observed for $n \mathrm{HEX}$, indicates a significant difference in sorption affinity compared to its branched isomers. The extension of the overshoot is more pronounced at higher pressure, and increases as the temperature decreases, i.e., the higher the coverage the higher the overshoot. Another interesting remark is the change in the adsorption hierarchy of the branched molecules at high pressure:

$\mathrm{T}=343 \mathrm{~K} \rightarrow 23 \mathrm{DMB}>3 \mathrm{MP}>22 \mathrm{DMB}$

$\mathrm{T}=373 \mathrm{~K} \rightarrow 23 \mathrm{DMB}>22 \mathrm{DMB}>3 \mathrm{MP}$

$\mathrm{T}=423 \mathrm{~K} \rightarrow 23 \mathrm{DMB}=22 \mathrm{DMB}>3 \mathrm{MP}$

$\mathrm{T}=473 \mathrm{~K} \rightarrow \mathrm{K} 22 \mathrm{DMB}>23 \mathrm{DMB}>3 \mathrm{MP}$

The effect of the temperature on the equilibrium selectivity, $S$, is shown in Fig. 4. Since equimolar mixtures are concerned, the equilibrium selectivity can be calculated by the ratio of the amount adsorbed, $q_{1} / q_{2}$, being component 2 the more adsorbed [11]. It can be seen from panel $a$ of Fig. 4 , that the selectivity between $n$ HEX and dibranched isomers is significant at low pressure. Interestingly, $S_{\mathrm{di}}$ branched/nHEx and $S_{\text {dibranched/3MP }}$ exhibit maxima at $423 \mathrm{~K}$. A different feature is observed at moderate pressure (panel $b$ of Fig. 4), where the selectivity exhibits a monotonic increase with the temperature.

At the same conditions, $S_{3 \mathrm{MP} / 22 \mathrm{DMB}}$ in UiO-66 is significantly lower than $S_{22 \mathrm{DMB} / 3 \mathrm{MP}}$ in zeolite beta [12]. For instance, at $473 \mathrm{~K}$ and $p_{\text {isom }}=6.3 \mathrm{kPa}, S_{3 \mathrm{MP} / 22 \mathrm{DMB}}=1.5$ in UiO-66 against 2.7 for zeolite beta. Thus, unlike zeolite beta, the UiO-66 does not make a significant discrimination between dibranched and monobranched $\mathrm{C}_{6}$ isomers.

According to the classical view on adsorption, the sorption selectivity is essentially governed by interaction energy. At low coverage, an adsorbed molecule is attracted by van der Waals forces towards the pore walls; the closer the molecules approaches the pore walls, the higher the intensity of the interactions. For instance, it is well known that the $n \mathrm{HEX}$ is preferentially adsorbed over its branched homologues on most zeolites because the configuration of the linear chain maximizes the contact surface area with the walls. The kinetic diameter $\left(d_{\mathrm{k}}\right)$, the molecular van der Waals surface $\left(S_{\mathrm{VDW}}\right)$ and the molecular van der Waals volume ( $\left.V_{\mathrm{VDW}}\right)$ are good measures of the degree of interaction between molecules and pore walls, and their variation as a function of the branching degree of the hexane isomers is represented in Table 2 . The values of $S_{\mathrm{VDW}}$ and $V_{\mathrm{VDW}}$ were computed by Hyperchem 8.0 software using the semi-empirical Geometry optimization AM1 (Austin Model 1) [15].

Unlike the general trend observed so far for typical microporous adsorbents, UiO-66 exhibits higher capacity for highly branched molecules. Generally, when dealing with structural isomers, the higher the branching degree, the higher kinetic diameter, and the lower the van der Waals volume. This tendency is clearly shown for hexane isomers in the data from Table 2. The preferential adsorption of branched alkanes over their linear counterparts has been experimentally observed by Santilli et al. [16] and Denayer et al. [17] in zeolite SAPO-5, and by Denayer et al. [8] in zeolite MCM-22. However, there are no examples in the literature for the inverse shape selectivity of long chain alkane isomers in MOF materials.

To better understand the inverse selectivity phenomena in UiO66, one should consider first, the structures SAPO-5 (AFI-type topology) and MCM-22 (MWW topology), in terms of pore geometry. The panel $a$ of Fig. 5 shows that the pore system in SAPO-5 consists of one-dimensional, straight cylindrical channels of diameter $0.73 \mathrm{~nm}$. This type of tubular pores only allows for the translational motion of the molecules. It was reported [17] that when a mixture of 22DMB, 2MP and nHEX is injected in a column packed with SAPO-5, the more retained component is the 22DMB, followed by the $n \mathrm{HEX}$ and the 2MP. The preferential adsorption of the dibranched isomer is explained by the higher packing efficiency of the shorter molecules, as illustrated in panel $a$ of Fig. 5. This behaviour is also called in the literature as length entropy effect, and was extensively addressed in molecular simulation studies [18-20].

Panel $b$ of Fig. 5 shows the supercavity in zeolite MCM-22, which consist of a larger central cavity, and two pockets located at the top and the bottom of the central cavity. The supercavity can be accessed through six apertures of dimensions $0.42 \times$ $0.51 \mathrm{~nm}$. In an experimental work, Denayer et al. [8] determined that in the MCM-22, the order of the sorption hierarchy in terms of loading is $3 \mathrm{MP}>n \mathrm{HEX}>22 \mathrm{DMB}$. The authors suggest that the short molecule 3MP adsorb preferentially in the pockets of the MCM-22 supercavity, where they maximize energetic interaction with the zeolite. On the other hand, longer molecule $n$ HEX is located in the larger central part of the supercavity, because their rotational motion in the pocket is restricted. The bulkier dibranched isomer 22DMB is less adsorbed, because it cannot access to the supercavities through the $0.42 \times 0.51 \mathrm{~nm}$ apertures. In zeolite MCM-22, the preferential adsorption of the monobranched alkane over its linear isomer is referred in the literature as a case of inverse shape selectivity based on the differences in rotational freedom in the pockets of the supercavity $[8,17]$. That is to say, the shape-selectivity mechanism is due to a rotational entropy effect.

The comparison between Fig. 1 and panel $b$ of Fig. 5 shows that the pore system of UiO-66 is, in a certain way, similar to the one of MCM-22. For instance, both structures possess two different types of cavities: the large ones have a diameter $1.1 \mathrm{~nm}$ and $1.6 \mathrm{~nm}$, in UiO-66 and MCM-22, respectively; the smaller ones have a diameter of $0.8 \mathrm{~nm}$ and $0.7 \mathrm{~nm}$, in UiO-66 and MCM-22, respectively. Such similarity, shown in Fig. 6, might explain the inverse selectivity observed in UiO-66. The main difference, in relation to MCM-22, is that the cavities can be accessed through windows 

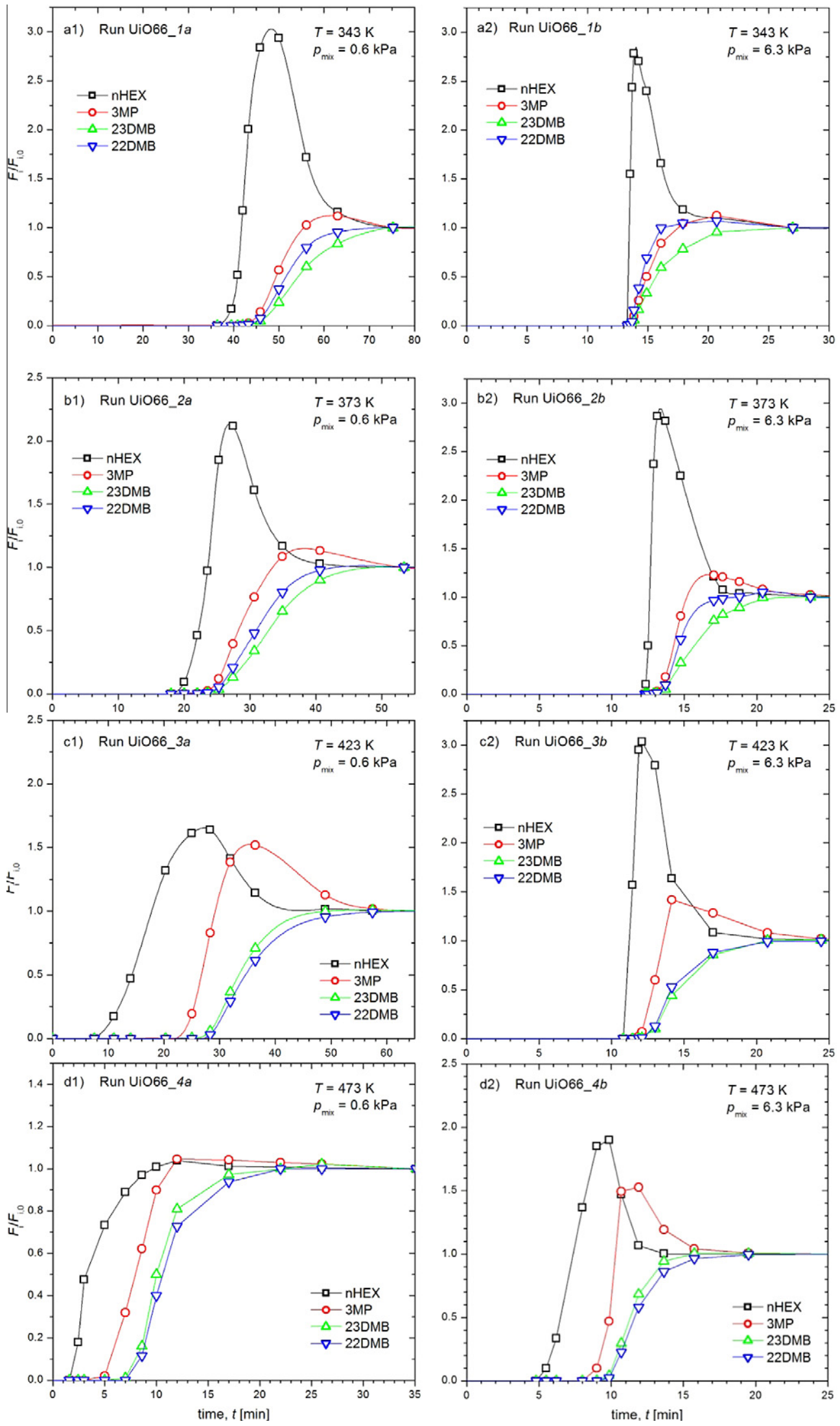

Fig. 3. Quaternary breakthrough curves of hexane isomers in UiO-66.

with a larger pore aperture up to $0.7 \mathrm{~nm}$. This particularity can explain the preferential adsorption of the bulkier dibranched hexane isomers in UiO-66. Moreover, the discussion about the retention of the $C_{6}$ isomers in the UiO-66 might also takes into account the 

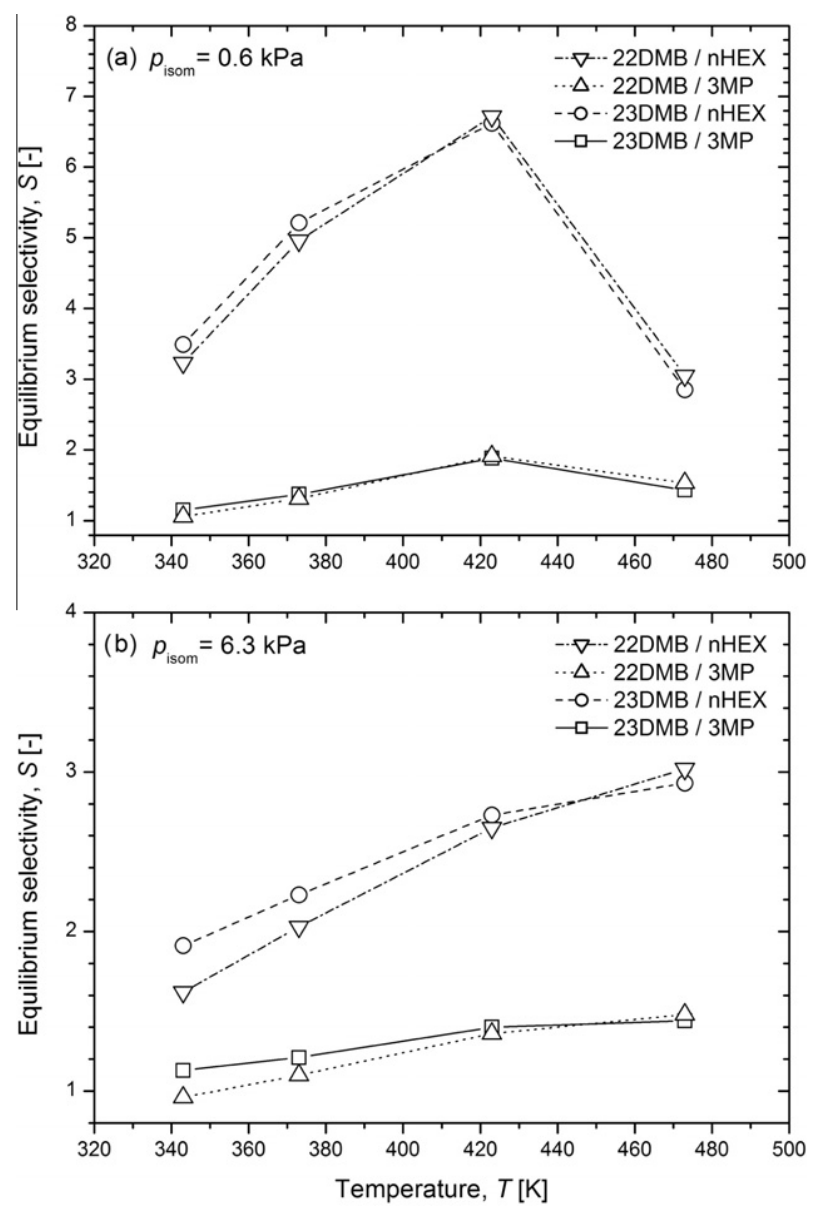

Fig. 4. Effect of the temperature in the equilibrium selectivity in MOF UiO-66 at a) $0.6 \mathrm{kPa}$, and b) $6.3 \mathrm{kPa}$.

molecular lengths reported in the literature [21] for $n \mathrm{HEX}$, $0.91 \mathrm{~nm}, 3 \mathrm{MP}, 0.86 \mathrm{~nm}$, and 22DMB, $0.67 \mathrm{~nm}$. These dimensions a

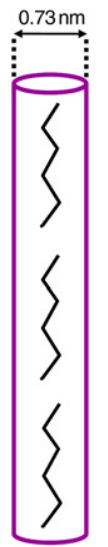

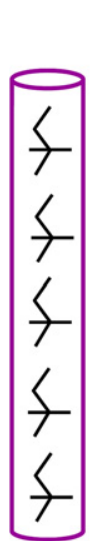

b

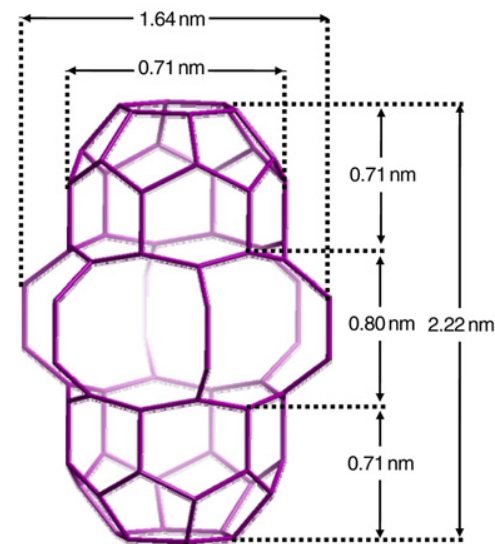

Fig. 5. Schematic representations: a) length entropy effect during adsorption of $n \mathrm{HEX}, 3 \mathrm{MP}, 22 \mathrm{DMB}$ and 23DMB in the cylindrical channels of AFI (adapted from [22]); b) MWW supercavity (adapted from [23]).

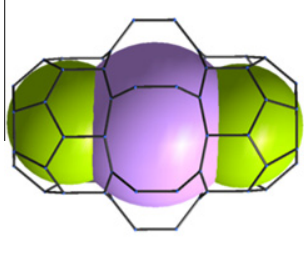

MCM-22

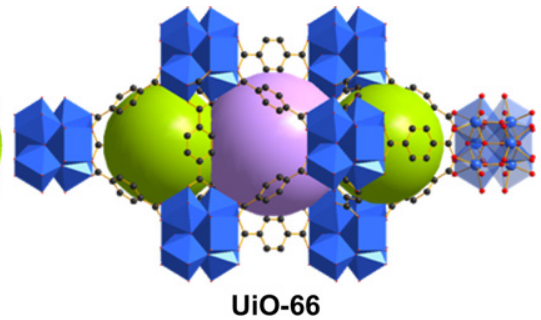

UiO-66

Fig. 6. View of the cavities of zeolite MCM-22 and MOF UiO-66.

are an indication that $n \mathrm{HEX}$ is too long to accommodate the small tetrahedral cavities, explaining in this way the distinct feature of its breakthrough curves in Fig. 3. On the other hand, the molecular length of the dibranched $C_{6}$ ensures that the molecule is short enough to rotate freely inside the small cavities. The theoretical support from molecular simulation data will be useful to clarify the

Table 2

Properties of hexane and xylene isomers.

\begin{tabular}{|c|c|c|c|c|c|}
\hline Component & Abbreviation & Structure & $d_{\mathrm{k}}(\mathrm{nm})$ & $S_{\mathrm{VDW}} \times 10^{2}\left(\mathrm{~nm}^{2}\right)$ & $V_{\mathrm{VDW}} \times 10^{3}\left(\mathrm{~nm}^{3}\right)$ \\
\hline$n$-hexane & $n \mathrm{HEX}$ & & $0.43^{\mathrm{a}}$ & 150.70 & 113.40 \\
\hline 3-methylpentane & 3MP & & $0.50^{\mathrm{a}}$ & 147.83 & 113.08 \\
\hline 2,3-dimethylbutane & 23DMB & & $0.56^{\mathrm{a}}$ & 145.41 & 112.66 \\
\hline 2,2-dimethylbutane & 22DMB & & $0.62^{\mathrm{a}}$ & 146.19 & 112.67 \\
\hline Ethylbenzene & $\mathrm{EB}$ & & $0.67^{\mathrm{b}}$ & 148.95 & 120.20 \\
\hline para-xylene & $p \mathrm{X}$ & & $0.67^{\mathrm{b}}$ & 148.92 & 120.13 \\
\hline meta-xylene & $m X$ & & $0.71^{\mathrm{b}}$ & 149.17 & 120.04 \\
\hline ortho-xylene & $o X$ & & $0.74^{\mathrm{b}}$ & 146.14 & 119.91 \\
\hline
\end{tabular}

\footnotetext{
a Data taken from [25].
}

b Data taken from [24]. 
adsorption phenomena in MOF UiO-66, as well as, to explain the curious maxima in the equilibrium selectivity shown in Fig. 4 a.

\subsection{Adsorption of quaternary mixtures of xylene isomers}

The adsorption behaviour of the xylene isomers in UiO-66 was investigated by performing a set of quaternary breakthrough curves, following the same experimental procedure used for the study of hexane isomers. The experiments were performed at $398 \mathrm{~K}$ and $423 \mathrm{~K}$, and hydrocarbon pressure of $1 \mathrm{kPa}$ and $10 \mathrm{kPa}$. The experimental conditions, and amount adsorbed are listed in Table 3.

The total adsorption capacity of UiO-66 for xylene isomers is practically twice of the one observed for hexane isomers (Table
3). Comparing with the physical properties of xylene isomers, shown in Table 2, it is clear that once again, the molecule with higher kinetic diameter and lower van der Waals volume $(o \mathrm{X})$ is the most adsorbed one. Fig. 7 depicts equimolar quaternary breakthrough curves of xylene isomers in UiO-66. These breakthrough curves clearly display the ability of UiO-66 to separate $o X$ from its homologues. In the experimental conditions studied, $p \mathrm{X}$ is always the less adsorbed molecule, while $o \mathrm{X}$ is from a far the more retained. Regarding the intermediate compounds, $m \mathrm{X}$ and $\mathrm{EB}$, the experiments indicate that their adsorption hierarchy is apparently influenced by the partial pressure.

Interestingly, UiO-66 cannot effectively discriminate between $p \mathrm{X}, m \mathrm{X}$, and EB. Moreover, a comparison between the retention times of theses isomers, and the one obtained for the $o \mathrm{X}$, suggests

Table 3

Experimental conditions for the equimolar quaternary breakthrough curves of xylene isomers in UiO-66.

\begin{tabular}{|c|c|c|c|c|c|c|c|c|}
\hline \multirow[t]{2}{*}{ Run } & \multirow[t]{2}{*}{$T(\mathrm{~K})$} & \multirow[t]{2}{*}{ Helium flowrate (ml/min) } & \multirow[t]{2}{*}{ Hydrocarbon pressure (kPa) } & \multicolumn{4}{|c|}{ Partial loading (wt.\%) } & \multirow[t]{2}{*}{ Total loading (wt.\%) } \\
\hline & & & & $\mathrm{pX}$ & $\mathrm{mX}$ & EB & $\mathrm{oX}$ & \\
\hline UiO66_1c & 398 & 40.0 & 1.0 & 2.91 & 3.26 & 3.69 & 5.34 & 15.20 \\
\hline UiO66_2c & 423 & & & 1.69 & 2.18 & 2.71 & 5.23 & 11.81 \\
\hline UiO66_1d & 398 & 7.0 & 10.0 & 3.05 & 4.93 & 4.04 & 8.68 & 20.70 \\
\hline UiO66_2d & 423 & & & 1.83 & 3.19 & 2.80 & 8.34 & 16.16 \\
\hline
\end{tabular}
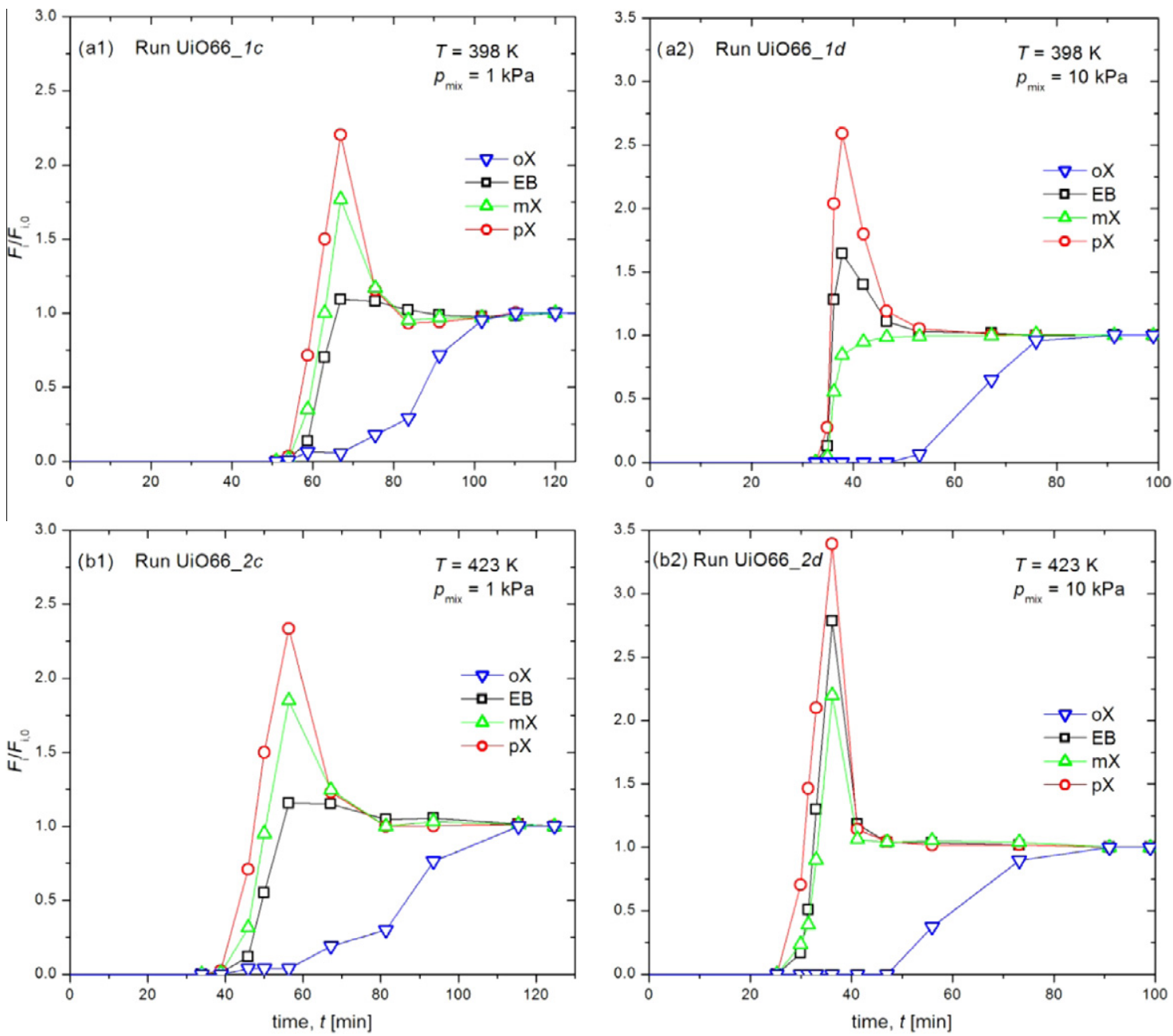

Fig. 7. Equimolar quaternary breakthrough curves of xylene isomers in UiO-66. 
that this latter is the only one that can be retained in both types of cavities. Apparently, the position of the methyl substituents in adjacent carbon atoms in the aromatic ring, might favour the rotation of the $o \mathrm{X}$ in the $0.8 \mathrm{~nm}$ cavities. Nevertheless, further inferences on the mechanism responsible for the ortho preference should be carefully taken, and requires the use of different approaches such as powder diffraction techniques or molecular simulation.

\section{Conclusion}

We present here the first experimental evidence of reverse shape selectivity for hydrocarbons in a MOF material. Multicomponent breakthrough experiments with hexane isomers and xylene isomers were addressed using MOF UiO-66 in the agglomerate form, at temperatures ranging from 343 to $473 \mathrm{~K}$, and partial pressures up to $10 \mathrm{kPa}$. The results obtained indicate that all the hexane and the xylene isomers can access to the large cavities in UiO-66. However, the selectivity towards bulkier isomers suggests that the retention in the small cavities is dictated by steric constraints.

Concerning the hexane isomers, it was shown that the sorption hierarchy is opposite to the one observed for typical adsorbents: $22 \mathrm{DMB}>23 \mathrm{DMB}>3 \mathrm{MP}>>n \mathrm{HEX}$. The distinct feature of the $n \mathrm{HEX}$ breakthrough curves is an indication that the linear chain is too longer to fit inside the small cavities. Relatively to xylene isomers, it was observed that adsorption of the bulkier oX is favoured over the other isomers. Apparently, the position of the methyl groups in the aromatic ring might play an important role in the retention of the xylene isomers in MOF UiO-66.

In a general way, the results suggest that the retention of structural isomers in UiO-66 is dictated by their rotational freedom in the small cavities.

\section{Reference}

[1] J.H. Cavka, S. Jakobsen, U. Olsbye, N. Guillou, C. Lamberti, S. Bordiga, K.P. Lillerud, J. Am. Chem. Soc. 130 (2008) 13850-13851.

[2] G. Férey, in: J. Cejka, H. van Bekkum, A. Corma, F. Schüth (Eds.), Introduction to Zeolite Science and Practice, Studies in Surface Science and Catalysis 168, Elsevier B.V, Amsterdam, 2007, pp. 327-374.

[3] G. Férey, Chem. Soc. Rev. 37 (2008) 191-214.

[4] O.M. Yaghi, M. O'Keeffe, N.W. Ockwig, H.K. Chae, M. Eddaoudi, J. Kim, Nature 423 (2003) 705-714.

[5] B. Smit, T. Maesen, Nature 451 (2008) 671-678.

[6] N.A. Cusher, in: R.A. Meyers (Ed.), Handbook of Petroleum Refining Processes, third ed., McGraw Hill, New York, 2004, pp. 29-39.

[7] A. Minkkinen, L. Mank, S. Jullian, US Patent 5233,120 1993.

[8] J.F.M. Denayer, R.A. Ocakoglu, I.C. Arik, C.E.A. Kirschhock, J.A. Martens, G.V. Baron, Angew. Chem. Int. Ed. (44) (2005) 400-403.

[9] J.F.M. Denayer, R.A. Ocakoglu, J. Thybaut, G. Marin, P. Jacobs, J. Martens, G.V. Baron, J. Phys, Chem. B 110 (2006) 8551-8558.

[10] L. Pan, D.H. Olson, L.R. Ciemnolonski, R. Heddy, J. Li, Angew. Chem. Int. Ed. 45 (2006) 616-619.

[11] P.S. Bárcia, J.A.C. Silva, A.E. Rodrigues, Ind. Eng. Chem. Res. 45 (2006) 43164328.

[12] P.S. Bárcia, J.A.C. Silva, A.E. Rodrigues, AIChE J. 53 (2007) 1970-1981.

[13] J.A.C. Silva, A.E. Rodrigues, AIChE J. 43 (1997) 2524-2534.

[14] A.P. Nelson, O.K. Farha, K.L. Mulfort, J.T. Hupp, J. Am. Chem. Soc. 131 (2009) 458-460.

[15] J.S. Dewar, G. Zoebisch, E. Healy, J.P. Stewart, J. Am. Chem. Soc. 107 (1985) 3902.

[16] D.S. Santilli, T.V. Harris, S.I. Zones, Microporous. Mater. 1 (1993) 329-341.

[17] J.F.M. Denayer, A.R. Ocakoglu, J.A. Martens, G.V. Baron, J. Catal. 226 (2004) 240-244.

[18] R. Krishna, B. Smit, S. Calero, Chem. Soc. Rev. 31 (2002) 185-194.

[19] R. Krishna, J.M. van Baten, Sep. Purif. Technol. 55 (2007) 246-255.

[20] M. Schenk, S. Calero, T.L.M. Maesen, T.J.H. Vlugt, L.L. van Benthem, M.G. Verbeek, B. Schnell, B. Smit, J. Catal. 214 (2003) 88-99.

[21] Y. Traa, S. Sealy, J. Weitkamp, Mol. Sieves 5 (2007) 103-154.

[22] B. Smit, R. Krishna, Chem. Eng. Sci. 58 (2003) 557-568.

[23] S. Huang, V. Finsy, J. Persoons, M.T.F. Telling, G.V. Baron, J.F.M. Denayer, Phys. Chem. Chem. Phys. 11 (2009) 2869-2875.

[24] M.P.M. Nicolau, P.S. Bárcia, J.M. Gallegos, J.A.C. Silva, A.E. Rodrigues, B. Chen, J. Phys, Chem. C 113 (2009) 13173-13179.

[25] M.L. Maloncy, L. Gora, J.C. Jansen, Th. Maschmeyer, Ars Separatoria Acta 2 (2003) 18-28. 